\title{
Profile of Malaria in Pregnant Women Attending Antenatal Clinics in Rural Community in Nigeria
}

\author{
B.E. Bassey*,1, J.E. Asor ${ }^{2}$ and M.F. Useh ${ }^{3}$ \\ ${ }^{1}$ Department of Medical Laboratory Services, Wuse General Hospital, P. O. Box 9011, Wuse, Abuja, Nigeria \\ ${ }^{2}$ Department of Zoology and Environmental Biology, University of Calabar, Calabar, Nigeria \\ ${ }^{3}$ Department of Medical Microbiology and Parasitology, University of Calabar
}

\begin{abstract}
We evaluated the malaria burden in randomly selected pregnant women (PW) attending antenatal clinics in Abuja, Nigeria, to establish an association between pregnancies, malaria. Structured questionnaire was administered by the ante-natal nursing staff and a research assistant. In total, 1400 pregnant women were screened between April and September 2004, and capillary blood samples were obtained and screened for malaria parasites in thin blood films and quantitative buffy coat analysis (QBC). In total, 1035 (73.9\%) pregnant women were positive for Plasmodium falciparum; of which 578 (55.8\%) were primigravidae; 299 (28.9\%) second gravidae; and 158 (15.3\%) were multigravidae, while 297 (28.7) were in their first trimester, $311(30 \%)$ were in their second trimester, and $427(41.3 \%)$ were in their third trimester. The highest prevalence of malaria parasite $(31.6 \%)$ was found in those aged 26-30 years while the lowest prevalence $(2.9 \%)$ occurred in those aged 41-46 years. Socio economically, prevalence of malaria parasite is highest in nonautomobile owners $84.4 \%, 66 \%$ in those with monthly income less than $\$ 100$, and those living in vegetable thatched houses had $46.0 \%$, while the lowest incidence (15.6\%) was found in automobile owners. Of the 760 pregnant women who sought malaria treatment only 278 (34.2\%) seek intervention in hospitals, while 59.4\% got treatment outside the hospital or were on self medication. This study demonstrated a high prevalence of malaria in the population evaluated, and therefore underlines the need for urgent intervention through capacity building, implementation of intermittent preventive treatment (IPT), use of insecticides treated-nets (ITN) and effective case management of malaria illness. The delivery of these interventions through ante-natal clinics in Nigeria is highly critical and needs to be encouraged; strategies that encourage pregnant women to attend antenatal clinics early and consistently need to be developed. It is also important to develop coherent and effective policies and tools to tackle malaria and poverty.
\end{abstract}

Keywords: Malaria, Rural-community, Nigeria, Pregnant women, Socio economic status.

\section{INTRODUCTION}

An estimated 30 million women living in malariaendemic areas of Africa become pregnant each year. Pregnant women are particularly vulnerable to malaria because pregnancy reduces immunity to malaria; increases susceptibility to malaria infection, the risk of illness, severe anaemia, acute pulmonary edema, renal failure, puerperal sepsis, postpartum haemorrhage, and increases the risk of death. Malaria in pregnancy results in adverse pregnancy outcomes, such as spontaneous abortion, neonatal death, and low birth weight. Chronic anemia, due to malaria may also affect a child's growth and intellectual development [1]. Malaria is preventable, yet a major cause of maternal and newborn illness and death in Africa, resulting in about 10,000 maternal deaths and between 75,000 and 200,000 infant deaths each year [1].

Several studies in endemic areas suggest that pregnant women have higher frequency of malaria, and are more likely to develop severe malaria when compared to their nonpregnant counterparts. Women in first and second pregnancies are noted to have higher parasitic concentration than others with greater than two pregnancies [2]. However, with successive pregnancies, the frequency and severity of the

*Address correspondence to this author at the Department of Medical Laboratory Services, Wuse General Hospital, P. O. Box 9011, Wuse, Abuja, Nigeria; Tel: 234-802-796-6265; E-mail: bassey69@yahoo.com disease is known to decline [2]. A cross-sectional study in an endemic area in Nigeria showed an increase in parasitic concentration, fever, inflammation, and anemia among primigravidae women [3]. P. falciparum was present in all but one infection, and gravidity influenced the occurrence of this particular parasite. Studies have also revealed an association between malaria infection rates and the period of pregnancy. An epidemiological study conducted in several countries in Africa, revealed an interesting pattern of the infection. Higher rates were observed during the first few weeks of pregnancy, which peaked during the second trimester. The rates declined in the last trimester and after pregnancy [2]. Another study carried out in the Gambia showed consistent decline in malaria antibody IgG as the pregnancy progressed [4].

Malaria is endemic in the poorest countries of the world and has often been labeled a disease of poverty. Several studies attempted to make the link between malaria and socio-economic status (SES) using a number of variables for measuring SES. Household income, occupation type, gender, and assets have all been used as proxies for assessing SES in many developing countries; these different measurement approaches make comparisons problematic. However, studies have shown that there is indeed an association between malaria occurrence and SES, as it relates to prevention and access to treatment of malaria [5]. Poverty level also influences the incidence of malaria. Malaria has also serious so- 
cio-cultural consequences in families by interfering with farm activities in the rainy season. It is estimated that malaria afflicted families on the average can harvest only $40 \%$ of the crops harvested by healthy families. Causes absenteeism from school, thus affecting school performance. It is estimated that in endemic areas like Uganda, malaria may impair as much as $60 \%$ of the schoolchildren's learning ability. Poor malaria-stricken family may spend up to $25 \%$ of income on malaria treatment and prevention, and loose household incomes through absenteeism from work. It is estimated that workers suffering from a malaria bout can be incapacitated for 5-20 days [6]. A study in Apac, Kampala, and Rukungiri Districts showed that malaria was responsible for $54 \%, 33 \%$ and $50 \%$ respectively of absenteeism from work per month in the above districts [6].

In Nigeria, malaria is associated with school absenteeism, loss of income, and man hour. The prevalence rate of malaria is 919 per 100,000 people with most $(80 \%)$ infected with $P$. falciparum [1]. In 1999, malaria hospital admissions in the country constituted $20 \%$ while outpatient attendance was $30 \%$ [7]. More recently, the rate of outpatient attendance due to malaria is estimated at about 50\% [7]. Due to the burden of malaria in the country, standard care of practice in antenatal clinics involves conducting a malaria parasitic count, and haemoglobin estimation. About $65 \%$ of pregnant women in the country receive antenatal care at least once, and almost $40 \%$ attend a second time [8]. However, few epidemiological studies have been conducted in malaria endemic areas in Nigeria to investigate specific factors associated with malaria in pregnancy. Yet, malaria transmission is increasing in Nigeria due to deforestation, cultivation of wetlands, poor environmental sanitation, other man made breeding sites such as construction works, brick pits or fish ponds, among others, all of which create breeding sites for Anopheles mosquito.

This study aimed to establish the relationship between malaria parasitaemia, pregnancy term, and number of pregnancies, age and socio-economic status of pregnant women. Information provided by this study would help to develop malaria control strategies in Nyanya, and surrounding villages, this area is of special interest because the population is exposed to $P$. falciparum.

\section{MATERIALS AND METOHOLOGY}

\section{Study Area and Population}

The study took place in Nyanya General Hospital, Abuja, Nigeria which is 8 kilometer from the city centre. Most pregnant women in the rural area of Nyanya receive antenatal care at the Nyanya General Hospital, a government funded facility, due to its proximity and the inexpensive services provided to the community. Predominantly, the inhabitants of Nyanya which has a total population of 1,405,201; Male 740,489 and Female 664, $712^{9}$ are civil servants and petty traders.

\section{Study Design}

One thousand four hundred (1400) pregnant women (PW) attending antennal clinic at Nyanya General Hospital were randomly selected for the cross sectional survey. The study lasted from April to September of 2004 during the rainy season, typically high malaria transmission period. A structured questionnaire was administered in English to de- termine malaria exposure and risk factors, assets, clinical malaria diagnosis (within the preceding 0-3 months, 4-6 months), febrile episodes, completion of malaria treatment, pregnancy number and term (first, second, and third), number of meals per day, anemia diagnosis, use of insecticides treated nets (ITN), house and latrine type, water source among others. Nurses at the antenatal clinic administered the questionnaire. The Ethical Committee of the hospital gave clearance in 2004, and all participants signed an informed consent after explanation of the procedure, the potential benefits, and absence of injury to participants.

\section{Parasite Count}

Thin blood smears were made from capillary blood anticoagulated in glass tubes containing ethylenediamine tetra acetate (EDTA). Each thin blood smear was made in duplicate, stained, and read independently at X100 magnification by two experienced microscopists unaware of the QBC (Quantitative Buffy Coat) tube test results. Parasitaemia was quantified by a modification of the method of Earle and Perez [9]. In brief, $10 \mu 1$ blood was spread evenly over a $10 \mathrm{x}$ $20 \mathrm{~mm}$ etched area of glass slide, the volume of blood per field was calculate, and parasite count estimated based on the number of parasites found and the number of fields examined.

Two hundred 200 field $(0.31 \mu 1$ blood $)$ were examined, and species of parasite determined by examination of the thin blood smears. The QBC tube is a capillary tube (Clay Adams Division, Becton Dickinson and Company, Franklin Park, New Jersey, USA) internally coated with acridine orange, potassium oxalate, sodium heparin, and EDTA. 55$65 \mu 1$ samples of venous blood transferred to the QBC tube by capillary action within 1 hour of collection. All tubes were immediately centrifuged in a QBC centrifuge at approximately $12000 \mathrm{~g}$ for 5 minutes and then placed upright. The QBC tubes were placed in acrylic holders, and the area surrounding the float just beneath the buffy coat examined under immersion oil. The entire circumference of the tube was examined systematically while moving away from the buffy coat through the erythrocyte layer. Parasite concentration was graded and recorded as $1+=1-4$ parasites per field, $2+=4-9$ parasites per field, $3+=$ more than 9 parasites per field.

\section{RESULTS}

All the 1,400 pregnant women approached willingly participated in the study. $1035(73.9 \%)$ were positive for malaria caused by $P$. falciparum, and $78(7.5 \%)$ were those aged 15-20 years. Results show that those aged 26-30 had the highest parasite burden of $327(31.6 \%)$, followed by 31 45 years $298(28.8 \%)$, while those $41-45$ years recorded the least parasite burden of $2.9 \%$. Salaried subjects recorded the lowest parasite burden of $162(15.7 \%)$, while unemployed subjects had the highest parasite burden 585 (56.5\%). Interestingly, results also show that married women harboured the highest parasite burden 1026 (99.1\%) compared to single women with $0.9 \%$ of total parasite burden. Educationally, secondary school leavers recorded high parasite burden 397 (38.4\%), primary school leavers 294 (28.4\%) while those who had no formal education recorded 208 (20.1\%). Those of Igbo ethnic extraction recorded the highest parasite burden of $251(24.3 \%)$ and the Gwaris recorded the least $P$. fal- 
ciparum burden of 128 (12.4\%). Respondents with monthly income of less than one hundred dollars recorded $66 \%$ of total malaria burden (Table 1).

Environmental factors such as type of housing affect exposure to malaria parasite. Study results show that more PW $476(46 \%)$ living in vegetable thatch houses, while the lowest malaria burden was found in those living in corrugated iron houses 197 (19\%). Similarly, the highest malaria burden was recorded in those living in mud houses $522(50.4 \%)$, while those living in houses with concrete wall had 513 (49.6\%). Interestingly, results show high parasite burden among in PW who do not own a car 874 (84.4\%). Surprisingly, those who used water closet toilet system recorded the highest burden of 574 (55.5\%), while those using pit latrines recorded 461 (44.5\%); High parasite burden was recorded in those who used well water $510(49.3 \%)$,and $115(15.1 \%)$ for those who used spring water. The use of insecticide treated nets (ITN) also influenced the outcome of malaria burden; those who did not sleep in ITN recorded the highest parasite

Table 1 Demographic Information on Pregnant Women Enrolled in the Study

\begin{tabular}{|c|c|c|}
\hline Risk Factors & No. Screened & Parasite Burden (\%) \\
\hline \multicolumn{3}{|l|}{ Age (years) } \\
\hline $21-25$ & 234 & $180(17.2)$ \\
\hline $26-30$ & 442 & $327(31.6)$ \\
\hline $36-40$ & 180 & $122(11.8)$ \\
\hline $41-45$ & 56 & $30(2.9)$ \\
\hline \multicolumn{3}{|l|}{ Employment Status } \\
\hline \multicolumn{3}{|l|}{ Marital Status } \\
\hline Married & 1356 & $1026(99.1)$ \\
\hline Single & 44 & $9(0.9)$ \\
\hline \multicolumn{3}{|l|}{ Education } \\
\hline None & 278 & $208(20.1)$ \\
\hline Primary & 388 & $294(28.4)$ \\
\hline Secondary & 528 & $397(38.4)$ \\
\hline \multicolumn{3}{|l|}{ Ethnicity } \\
\hline Igbo & 312 & $251(24.3)$ \\
\hline Yoruba & 284 & $198(19.1)$ \\
\hline Others & 339 & $218(21.0)$ \\
\hline \multicolumn{3}{|l|}{ Monthly income } \\
\hline \multicolumn{3}{|l|}{ Respondent } \\
\hline$<\$ 100$ & 752 & $683(66.0)$ \\
\hline$>\$ 100$ & 648 & $352(34.0)$ \\
\hline
\end{tabular}


burden 814 (78.6\%), while $21.4 \%$ of PW slept in ITNs (Table 2).

In understanding the relationship between pregnancy, malaria episodes, and malaria parasites concentration, Results show that first pregnancy had the highest parasite concentration 578 (55.8\%), second pregnancy, 299 (28.9\%), and ,$\geq 3$ pregnancies recoded the least parasite concentration. . In relation to pregnancy term, those in the third trimester recorded the highest parasite concentration, 427 (41.3\%), followed by those in their second trimester $311(30 \%)$ and those in their first trimester recorded the lowest parasite concentration of $297(28.7 \%)$. PW who did not report malaria episode within the preceding 0-3 months, 4-6 months recorded high parasite concentration $519(57.1 \%)$, while those who had reported episode of malaria recorded 444 (42.9\%), and those who indulged in self medication had parasite burden of 482 $(59.4 \%)$ (Table 3).

\section{DISCUSSION AND CONCLUSION}

Two-third of pregnant women in sub-Saharan Africa attend ante natal clinics at least once during pregnancy, an opportunity to provide them with health education, counseling, and intermittent preventive treatment, to prevent malaria [10]. Malaria is an increasing problem especially in Africa, in terms of disease burden, low socio-economic status, and

Table 2. Environmental Factors Associated with Malaria Status of Respondents

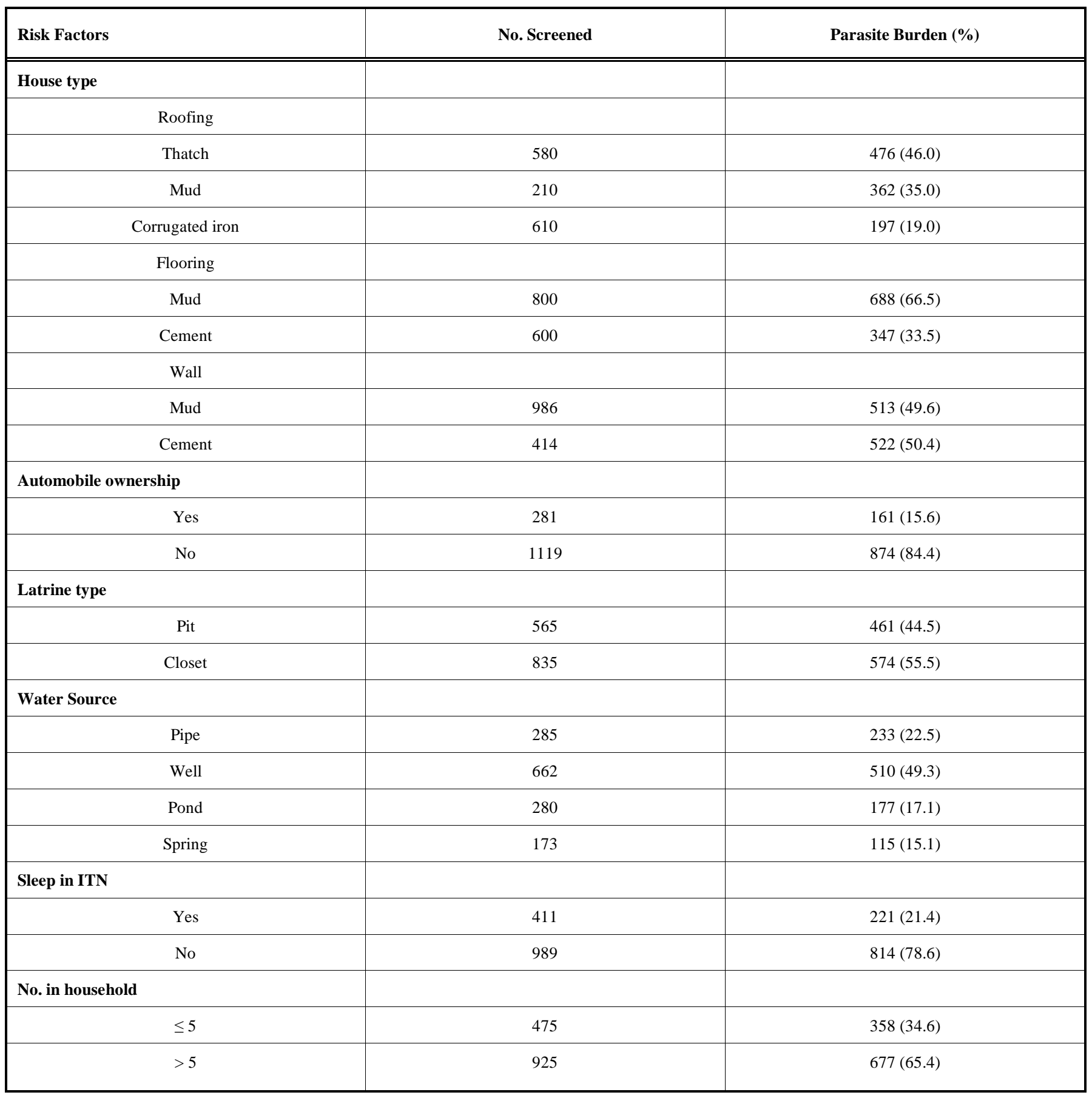


Table 3 Pregnancy Status, Malaria Episode and Associated Parasite Concentration

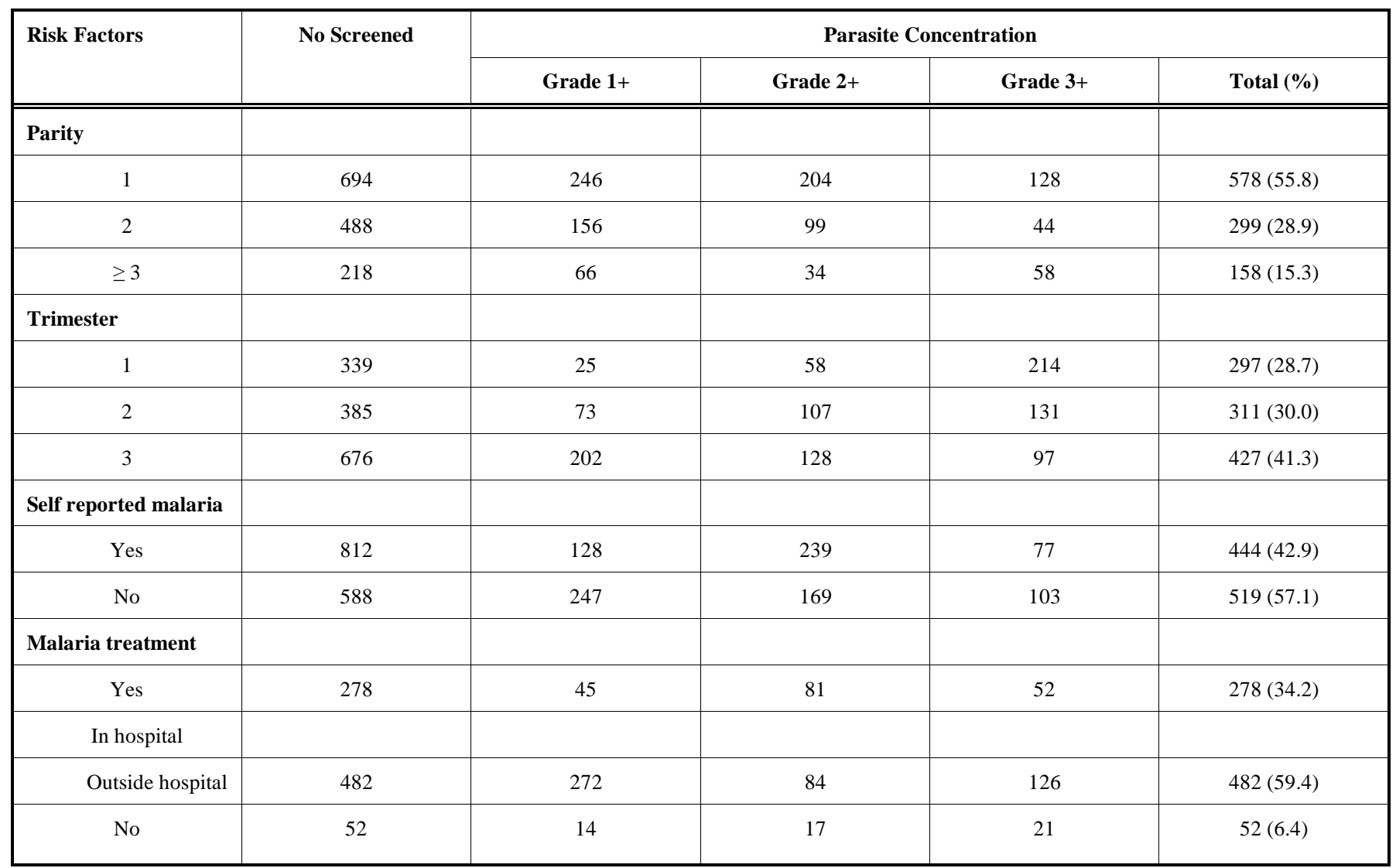

resistance to conventional therapy [2]. This may be attributed to poverty, drug resistance and environmental factors [2].

This study aimed to understand the prevalence and predictors of malaria parasitaemia, in pregnancy among women in Nyanya rural community in Nigeria during one malaria transmission season. Variables examined are age, term of pregnancy, number of pregnancies, the use of ITN, nature of housing, source of water, and others. The study also examined similarities between clinically diagnosed malaria and self reported malaria and fever episodes.

Results show that the prevalence and intensity of malaria varied considerably between pregnancy term, parity, and occupation, use of ITNs and socio-economic status of pregnant women screened. Our study revealed that 1035 (73.9\%) of the 1400 pregnant women screened had detectable $P$. falciparum in their peripheral blood sample. This finding (73.9\%) is slightly higher than those of Kisumu $95(51.1 \%)$ of the $186 \mathrm{PW}$ screened; and $62(40.5 \%)$ of the $153 \mathrm{PW}$ screened in Mombasa, Kenya [11]; 67\% recorded in central India [12] and 60\% recorded in Lagos, Nigeria [3]. Even much higher than the $36.2 \%$ recorded in another study conducted in Jos, Bauchi and Eku [13]. The reason for the high outcome recorded in this study may be attributed to the use of QBC technique which is more sensitive than the direct smear technique, large sample size and varying transmission pattern [14].

The highest malaria prevalence was among primigravidae $(55.8 \%)$, then second gravidae $(28.9 \%)$ and multigra- vidae $(15.3 \%)$, which accords with findings in India; primigravidae $(23 \%)$, second gravidae $(17 \%)$ and $11 \%$ in multigravidae [12]. The prevalence of $P$. falciparum among PW was highest in the first trimester $41.3 \%$, and decline to $30.0 \%$ and $28.7 \%$ in the second and third trimesters respectively. Results of this study are not in agreement with reports of similar prevalence's of $P$. falciparum parasitaemia in other places [12], but consistent with findings in Chandigarh, Northern India, which report highest parasite concentration in the third trimester and parity which had no influence in parasite concentration [15]. This trend may be attributed to physiological status of the PW and variations prevailing environmental factors.

Treated nets (ITNs) had a favorable impact on the prevention of malaria [16]. Results of this study supported by those of other studies on the relationship between the use of ITNs and parasite concentration. This study shows reduction in parasite concentration among users of ITNs from $78.6 \%$ to $21.4 \%$, this is in agreement with the findings of a similar study in Kabompo, Zambia which recorded a drop in parasite concentration from $38.1 \%$ to $7.7 \%$ [17], an indication that sleeping in ITNs helps to improve pregnancy outcome. In Gambia, studies also found that the use of ITNs reduced the overall risk of mortality and morbidity among pregnant women [16]; Further evidence comes from another study in a highly malarious area of Kenya, in which first four pregnancies in PW protected by ITNS gave birth to $25 \%$ fewer premature babies than their counterpart who did not sleep under ITNs [18]. 
Results of this study indicate that house type influenced the incidence of malaria among PW screened, as high incidence was observed in those living in vegetable thatched houses, as well as mud walls and floors. This suggests that thatch roof provides easy passage and hiding place, while mud wall and floor cracks and cavities equally serve as hiding places for mosquitoes. Furthermore, vegetable thatched roofs may not provide enough retention of insecticide spray.

The number of people living in a household also influenced parasite concentration, higher parasite concentration was observed in household with more than 5 persons, and lower parasite concentration in household with less than 5 persons. This may be attributed to overcrowding, poverty, and frequency of the number of times household doors are opened which increases the volume of mosquitoes that gain access to the house. The significant role economic factor played in malaria burden is demonstrated in the high incidence of P. falciparum in PW whose monthly income was less than one hundred dollars. This current trend associated with the economic status of PW reflects primarily distinct risk profiles across lifestyles and environmental factors, which provides favorable condition for the spread of malaria infection.

There was significantly high incidence in users of pit latrine; the reason for this trend may be attributed to the fertile breeding site provided by pit latrines.

The SES indicators used, (number of people in the participants' homes and house and automobile ownership) were not completely adequate in assessing SES status in the area. Only a few people in the Nyanya area owned cars or houses, $20.1 \%$ of the women surveyed. Perhaps a larger sample may have identified a noteworthy association. The major limitation in this study is the timing of antenatal visits by pregnant women; more women visited the clinics late in their pregnancy rather than early. This may have underestimated the risk of malaria infection which is typically seen among women earlier in their pregnancy [2]. Data for this study were collected only once during the rainy season, a repeated survey approach would have captured seasonal variations and their effect on the acquisition of the malaria parasite and might have increased the strength of the results. Response to culturally sensitive questions such as age and pregnancy term may have affected the inferences made in this study by the presence of others in the waiting area. Many in the Nyanya area believe that announcing the length of pregnancy is bad luck and could disrupt the natural pregnancy progression leading to miscarriage or stillbirth.

Finally, results of this study show high prevalence of $P$. falciparum infection in PW seen in the ante-natal clinic in Nyanya despite the periodic intermittent preventive treatment (IPT) that started in year 2000 as recommended by World Health Organization for the prevention of malaria in PW. Insufficient data in the performance of IPT in Nigeria supports the recommendation and imperative to develop strategic framework for malaria control with a data based component. Health professionals should be encouraged to in- clude IPT as part of ante natal care, education of women of childbearing age about the dangers of malaria in pregnancy and the potential benefits available to expectant mothers who use ITNs, make early and consistent attendance at ante natal clinics.

This study is relevant in rural communities such as Nyanya, where the standard of living in the area is low. No such studies have been done in the area; therefore, this study provides preliminary information to future research and potential interventions in the area. This study results indicates an increase risk of clinical malaria early in pregnancy, so an intervention encouraging pregnant women to visit the antenatal clinics early in pregnancy may significantly diminish the burden of the infection among pregnant women in malaria endemic areas.

\section{REFERENCES}

[1] The African Submit on Roll Back Malaria, Abuja, Nigeria, 25 April WHO/CDS/RBM/2000.17.

[2] Brabin BJ. An Analysis of Malaria in Pregnancy in Africa. Bull World Health Organization 1983; 61(6):1005-16.

[3] Omolade OO. The Status of Malaria among Pregnant Women: A study in Lagos Nigeria. Afr J Reprod Health 2003; (3): 77-83.

[4] McGregor IA, Rowe DS, Wilson ME. Plasma immunoglobulin concentrations in an African Gambian community in relation to season, malaria and other infections and pregnancy. Clin Exp Immunol 1970; $7: 51$

[5] Worrall, SB, Hanson K. The relationship between socio-economic status and malaria: a review of the literature. M.Sc. diss, Dept of Public Health, London School of Hygiene Tropical Medicine 2003.

[6] Ministry of Health. The burden of malaria in Uganda: why all should join hands in the fight against malaria MOH-MLA-12. 2001.

[7] Lamikanra OT. A Study of Malaria Parasitaemia in Pregnant Women, Placenta, cord blood and newborn babies in Lagos, Nigeria. West Afr J Med 1993; 12:213-217.

[8] Demographics and Health Survey. www.measuredhs.com 1999.

[9] Earle WC, Perez M. Enumeration of parasites in the blood of malarial patients. J Lab Clin Med 1932; 17: 1124-30.

[10] National Population Commission. Federal Republic of Nigeria Official Gazette no. 4, 2007; Vol. 94.

[11] Praise ME, Lewis SL, Ayisi GJ. A Rapid Assessment approach for Public Health decision making related to the prevention of Malaria during Pregnancy. Bull World Health Organization 2003; 81: 316323.

[12] Singh N, Shukia MM, Valecha N. Epidemiology of Malaria in Pregnancy in central India. Bull World Health Organization 1999; 77(7): 567-572.

[13] Egwunyenga AO, Ajayi JA, Nmorsi OPG, Duhlinska Popova DD. Plasmodium/intestinal Helminth co-infection among pregnant $\mathrm{Ni}$ gerian women. Mem Inst Oswaldo Cruz 2001; 96(8): 1055-1059.

[14] Leland R, Gary WL, Richard O, Arturo C. Rapid diagnosis of malaria by acridine orange staining of centrifuged parasites. Lancet 1989; 14: 68-71.

[15] Sholapurker SL. Malarial parasite concentration in infected pregnant women from Northern India. J Med Res 1988; 88: 228-230.

[16] D' Alessandro U. The impact of a national impregnated bed net programme on outcome of pregnancy in primigravidae in the Gambia. Trans Roy Soc Trop Med Hyg 1996; 90 (5): 487-492.

[17] Mclean KL, Senthilselvan A. Mosquito bed nets: implementation in rural villages in Zambia and the effect on sub clinical parasitaemia and haemoglobin. Trop Doct 2002; 32: 139-142.

[18] Kuile T. Permethrin-treated bed nets reduce malaria in pregnancy in an area of intense peremial malaria transmission in western Kenya. Am J Trop Med hyg 2003; 29:1181-9. 